\title{
Spectral-decomposition techniques for the identification of periodic and anomalous phenomena in radon time-series
}

\author{
R. G. M. Crockett ${ }^{1}$, F. Perrier ${ }^{2,3}$, and P. Richon $^{4,5}$ \\ ${ }^{1}$ School of Science and Technology, University of Northampton, NN2 6JD, UK \\ ${ }^{2}$ Équipe de Géomagnétisme, Institut de Physique du Globe de Paris, UMR-7154, 4 place Jussieu, 75005 Paris, France \\ ${ }^{3}$ Université Paris Diderot, 4 place Jussieu, 75005 Paris, France \\ ${ }^{4}$ CEA, DAM, DIF, 91297 Arpajon, France \\ ${ }^{5}$ Équipe de Géologie des Systèmes Volcaniques, Institut de Physique du Globe de Paris, UMR-7154, 4 place Jussieu, \\ 75005 Paris, France
}

Received: 29 November 2009 - Revised: 8 February 2010 - Accepted: 5 March 2010 - Published: 24 March 2010

\begin{abstract}
Two hourly-sampled time-series of soil-gas radon concentrations of durations of the order of a year have been investigated for periodic and anomalous phenomena. These time-series have been recorded in locations having little or no routine human behaviour and thus are effectively free of significant anthropogenic influences. One measurement site, Sur-Frêtes, is located in the French Alps, with saturated soil conditions; the second site, Syabru-Bensi, is located in $\mathrm{Nepal}$, in a river terrace with unsaturated soil conditions. In such conditions, periodic components with periods ranging from $8 \mathrm{~h}$ to 7 days are often weak and intermittent and therefore, even in the presence of stationary forcing, difficult to identify.

Two spectral decomposition techniques, Empirical Mode Decomposition (EMD) and Singular Spectrum Analysis (SSA), have been applied to these time series and yield similar results. For Sur-Frêtes, weak diurnal and semi-diurnal components are observed with EMD, while SSA reveals only a diurnal component. In Syabru-Bensi, both EMD and SSA reveal a strong diurnal component and a weaker semi-diurnal component. Tidal components M1 and M2 are also suggested by EMD in Sur-Frêtes, while these frequencies are not observed in Syabru-Bensi. The development of such analytical techniques can help in characterising the multiple physical processes contributing to the surface and subsurface dynamics of soil gases.
\end{abstract}

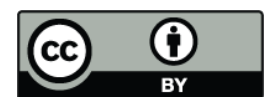

Correspondence to: R. G. M. Crockett (robin.crockett@northampton.ac.uk)

\section{Introduction}

Analysing time-series of radon emissions and concentrations for the presence of anomalies and/or cycles has the potential to reveal important information regarding crustal and surficial structures and processes, e.g. location and behaviour of faults, response to tidal forces and changes in stresses associated with earthquakes. However, the analysis is complicated by the stochastic nature of radon emissions and the range of natural and anthropogenic influences these are susceptible to (e.g. Crockett et al., 2006a; Miles, 2001; Neves et al., 2009).

Building on independent investigations by research groups in France, and the University of Northampton, UK, hourlysampled radon time-series of durations of the order of a year have been investigated for periodic and anomalous phenomena. We present results for two time-series of radon concentration in the soil from two different sites. The first time series, the central one-year period from longer time-series of 977 days total duration, has been recorded at the SurFrêtes ridge in the French Alps at a depth of $80 \mathrm{~cm}$ (Perrier et al., 2009a). The soil was assumed to be always saturated at that location. The second time series, of 336 days duration, has been obtained in the soil of a river terrace, at a depth of $30 \mathrm{~cm}$, in Syabru-Bensi, Nepal, in the vicinity of a geothermal zone (Perrier et al., 2009b). This site is characterised by a dry winter season and a summer season dominated by heavy monsoon rains. The shallow soil at this site is potentially affected by large seasonal effects and unsaturated conditions. These two locations have no routine human behaviour and thus are effectively free of significant anthropogenic influences. 
Initially, Fourier and Maximum Entropy techniques were used. However, the stochastic nature of radon emissions from rocks and soils, coupled with sensitivity to a wide variety of influences such as temperature, wind-speed and soil moisture-content has made interpretation of the results thus obtained difficult and only partially conclusive. Indeed, while some external forces such as tidal influences are fundamentally stationary, the radon response from the soil or bedrock can be highly non-linear (Richon et al., 2009), and result in non-stationary variations. Harmonic components in such geophysical time series, when present, are expected to be affected by significant temporal modulations. In essence, these time-series contain relatively small stationary features, i.e. approximately diurnal and semi-diurnal cycles, masked by relatively large non-stationary features, e.g. meteorological influences.

Thus, it was decided to investigate the use of spectral decomposition techniques, specifically Empirical Mode Decomposition (EMD) and Singular Spectrum Analysis (SSA). These techniques, in variously separating aperiodic and "high", "middle" and "low" frequency periodic components, effectively "de-noise" the data by allowing components of interest to be isolated from others which (might) serve to obscure (weaker) information-containing components. Once isolated, these components can be investigated using a variety of conventional techniques.

In this investigation, these spectral decomposition methods have been used successfully to indicate the presence of diurnal and sub-diurnal cycles in radon concentration which we provisionally attribute to solid-earth and barometric tidal influences. Also, these methods have been used to enhance the identification of short-duration anomalies in radon timeseries, attributable to a variety of causes including, for example, earthquakes and rapid large-magnitude changes in weather conditions (Crockett and Gillmore, 2010).

\section{Empirical Mode Decomposition and Singular Spectrum Analysis}

The following descriptions are outlines of the basic principles and are not substitutes for the indicated fuller mathematical descriptions and considerations.

\subsection{Empirical Mode Decomposition}

For a fuller description see, for example, Huang et al. (1998). In brief, EMD considers a signal to comprise a set of layers (Intrinsic Mode Functions, IMFs), each determined according to frequency content, built onto an aperiodic underlying state (the Residual). In operation, it iteratively identifies, "sifts", the IMFs from highest to lowest frequency content until no further IMFs can be identified and the Residual is obtained. Thus, for initial data (time-series assumed for con- venience), $T_{0}(t)$, the output of the first iteration is $T_{1}(t)$ and at a general $i^{t h}$ iteration, i.e. $T_{i-1}(t) \rightarrow T_{i}(t)$, EMD:

i) identifies the local maxima and minima in the input data $T_{i-1}(t)$

ii) from these, interpolates the separate maximum and minimum envelopes, $\operatorname{Max}_{i}(t), \operatorname{Min}_{i}(t)$ (cubic-splining is generally but not necessarily used for the interpolation);

iii) from these, calculates the mean locus which is the iteration-residual, $R_{i}(t)$ i.e. $R_{i}(t)=$ $\frac{1}{2}\left(\operatorname{Max}_{i}(t)+\operatorname{Min}_{i}(t)\right)$

iv) from these, calculates the iteration-IMF, $I_{i}(t)$, as the data minus the residual $I_{i}(t)=T_{i-1}(t)-R_{i}(t)$ and so $T_{i-1}(t)=I_{i}(t)+R_{i}(t)$

v) the residual $R_{i}(t)$ is either

a) passed as the input to the next, $(i+1)^{t h}$, iteration as $T_{i}(t)=R_{i}(t)$, or

b) becomes the overall Residual at final iteration $R(t)=R_{i}(t)$

vi) at the final iteration, for a total $n$ iterations, $R(t)=$ $R_{n}(t)$ and

$$
T_{0}(t)=I_{1}(t)+I_{2}(t)+\ldots+I_{n}(t)+R(t) .
$$

Thus, at each iteration, the highest frequency component in the data is "sifted" as the IMF. During an EMD process, the IMFs have progressively lower-frequency content and, because the process is empirical and does not assume any timefrequency structure in the data, any individual IMF will be more or less frequency-homogeneous depending on the data undergoing the decomposition process. Depending on the time-frequency characteristics of the data, it might be necessary to consider the sum of two or more adjacent IMFs to obtain a complete description of any given frequency component in the data. The EMD library for $\mathrm{R}$ (statistical language) was used for this investigation (Kim and Oh, v1.2, 2008).

\subsection{Singular Spectrum Analysis}

Singular Spectrum Analysis (SSA) is a Principal Components Analysis (PCA) technique in which the set of input vectors comprises a time-series and phase-lagged copies of itself. For a full description and theoretical consideration see, for example, Ghil et al. (2002), Vautard and Ghil (1989): the following summary description presents the basic principles of the technique with reference to PCA.

In brief, SSA decomposes a signal in terms of phaselagged versions of itself up to a user-selected maximum lag, for which there are constraints (Ghil et al., 2002). Given an $n$-element time-series, $T(t)=T(0), T(1), T(2), \ldots, T(n-1)$, and maximum lag $l$, the set of $l$ input vectors, all of length $(n+(l-1))$ comprises: 


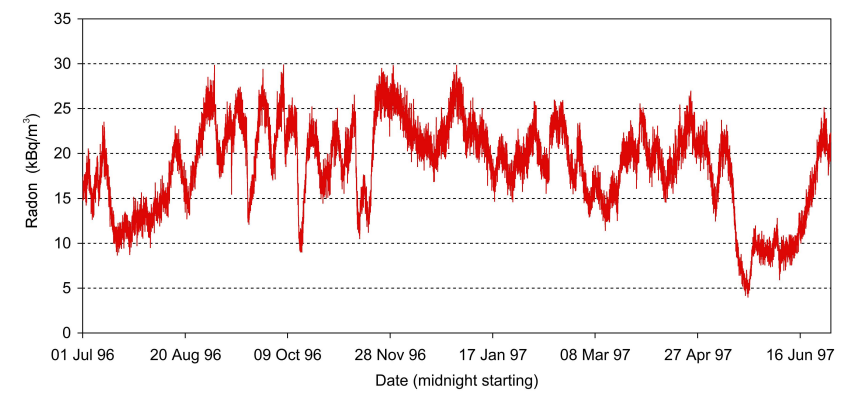

Fig. 1. Time-series of the Sur-Frêtes data.

$v_{1}(t)=T(t)$ padded with $(l-1)$ zeros at the end

$v_{2}(t)=T(t-1)$ padded with 1 zero at the start and $(l-2)$ zeros at the end

$v_{3}(t)=T(t-2)$ padded with 2 zeros at the start and $(l-3)$ zeros at the end

:

$v_{l}(t)=T(t-(l-1))$ padded with $(l-1)$ zeros at the start.

Thus, the set of input vectors can be considered as an embedding of the time-series in an $(n+(l-1)) \times l$ Toeplitz matrix having upper and lower triangles of zeros. This matrix increments time down the columns (row index) and phase-lag along the rows (column index).

From here, the covariance and diagonalisation matrices and the principal components are obtained. Note that the principal components have length $(n+(l-1))$ corresponding to the length of the input vectors in which the timeseries has been embedded. Each element of the covariance matrix depends on the phase-lag between two input vectors (i.e. columns of the Toeplitz matrix) and the variation of the covariance with lag can be considered in the same way as the variation of the correlation coefficient when performing lagged autocorrelation.

A reconstructed (spectral) component (RC) can be obtained from the desired principal component by repeating the above embedding process with the particular principal component (in place of the time-series). The artificially introduced phase-lag must be reversed in order to reconstruct a spectral component and this can be done by reversing the column-order of the Toeplitz matrix before "de-diagonalising" to obtain the reconstructed vector. This column-reversed matrix has dimension $(n+2(l-1)) \times l$ and thus the reconstructed vector has length $(n+2(l-1))$, containing the $n$-element reconstructed component padded by $(l-1)$ zeros at each end.

SSA determines the significance of (spectral) components according to covariance and, thus, the ranking of the components is not determined according to frequency. There are

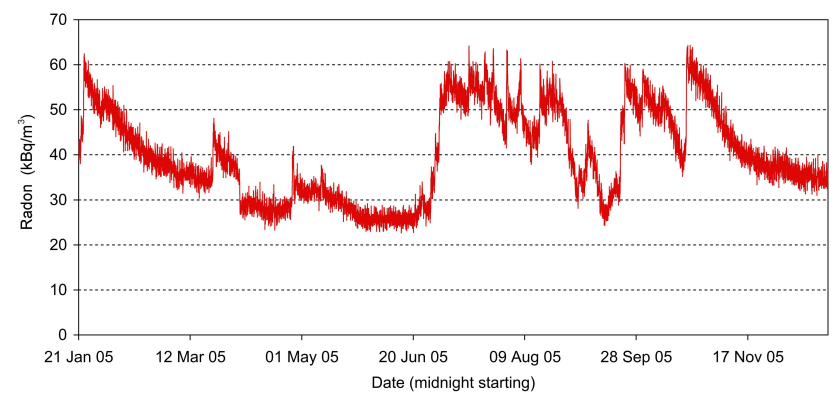

Fig. 2. Time-series of the Syabru-Bensi data.

various criteria for determining which components are significant and which are not (Ghil et al., 2002) but these do not completely eliminate the possibility of examining many low ranking components when investigating a time-series for weak cyclic/periodic features. SSA code written by the authors in Scilab was used for this investigation.

\section{Results}

Figure 1 shows the central one-year period, 1 July 1996 to 30 June 1997, for the Sur-Frêtes time-series (Perrier et al., 2009a); Fig. 2 shows the Syabru-Bensi time-series, 21 January 2005 to 22 December 2005 (Perrier et al., 2009b). In each case, non-stationary features are clearly visible in the data, some having magnitudes exceeding those of the stationary features under investigation. Empirical Mode Decomposition of the time-series yielded 12 IMFs in each case, with broadly similar diurnal and sub-diurnal frequency components. In both cases, IMF-1 comprises high-frequency "sampling" noise; IMF-2 contains frequency spectra centred at 4 cycles per day, having little consistent distinct structure; IMF-3 contains distinct frequency components centred at 2 cycles per day; IMF-4 contains distinct frequency components centred at 1 cycle per day and IMFs 5-12 comprise intermittent low-frequency quasi-periodic components.

Figures 3 and 4 show spectrograms for Sur-Frêtes IMFs 3 and 4 respectively. Figure 5 shows the spectrogram for Syabru-Bensi IMFs 3 and 4 combined, this being possible because of the tighter groupings of the frequencies in the individual IMFs and also the greater similarity in amplitude of these IMFs compared to the corresponding Sur-Frêtes IMFs. All spectrograms were performed using $3000 \mathrm{~h}$ (125 day) Hanning windows moved forward at $120 \mathrm{~h}$ (5 day) intervals. The results are summarised in Table 1.

Singular Spectrum Analysis of both time-series was performed using a 30-h window, this being sufficiently long to ensure that diurnal and semi-diurnal frequency components are revealed. For the Sur-Frêtes time-series, SSA revealed four significant components, of which the first two comprise major (non-stationary) features of the data and the third and fourth contain frequency spectra centred at 1 cycle per day. 
Table 1. Summary of Results of EMD and SSA with the two time series.

\begin{tabular}{|c|c|c|c|}
\hline & & $\begin{array}{l}\text { Sur-Frêtes, France. } \\
\text { (Perrier et al., 2009a) }\end{array}$ & $\begin{array}{l}\text { Syabru-Bensi, Nepal } \\
\text { (Perrier et al., 2009b) }\end{array}$ \\
\hline Location & & $45.705^{\circ} \mathrm{N} ; 6.651^{\circ} \mathrm{E}$ & $28.163^{\circ} \mathrm{N} ; 85.252^{\circ} \mathrm{E}$ \\
\hline Time reference & & UTC & Local (UTC+5.75) \\
\hline Soil condition & & saturated & unsaturated \\
\hline Probe depth & & $0.8 \mathrm{~m}$ & $0.3 \mathrm{~m}$ \\
\hline \multirow[t]{4}{*}{ Semi-diurnal } & & Fig. 3: EMD IMF 3 & Fig. 5: EMD IMFs 3,4 \\
\hline & & & Fig. 7: SSA RCs 3-6 \\
\hline & $2 \mathrm{cpd}, 12-\mathrm{h}, \mathrm{S} 2$ & strong evidence & strong evidence \\
\hline & $1.93 \mathrm{cpd}, 12.4-\mathrm{h}, \mathrm{M} 2$ & weak evidence & \\
\hline \multirow[t]{3}{*}{ Diurnal } & & Fig. 4: EMD IMF 4 & Fig. 5: EMD IMFs 3,4 \\
\hline & & Fig. 6: SSA RCs 3,4 & Fig. 7: SSA RCs 3-6 \\
\hline & $\begin{array}{l}1 \mathrm{cpd}, 24-\mathrm{h}, \mathrm{S} 1 \\
0.97 \mathrm{cpd}, 24.8-\mathrm{h}, \mathrm{M} 1\end{array}$ & $\begin{array}{l}\text { strong evidence } \\
\text { clear evidence }\end{array}$ & strong evidence \\
\hline
\end{tabular}

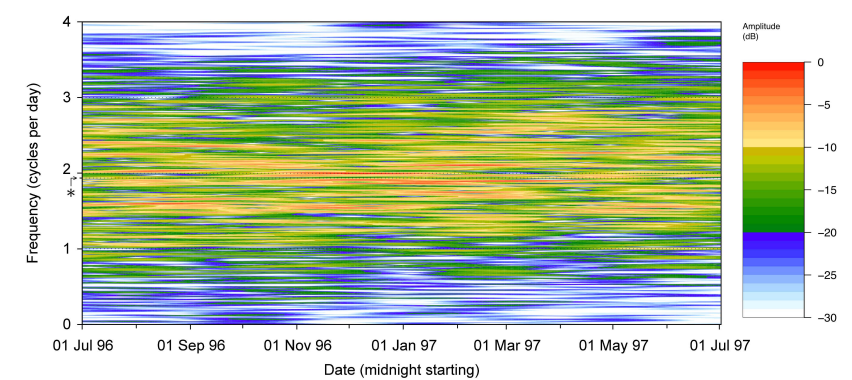

Fig. 3. EMD, Sur-Frêtes, IMF 3; frequencies grouped around 2 cycles per day (the asterisk indicates the M2 tidal harmonic frequency).

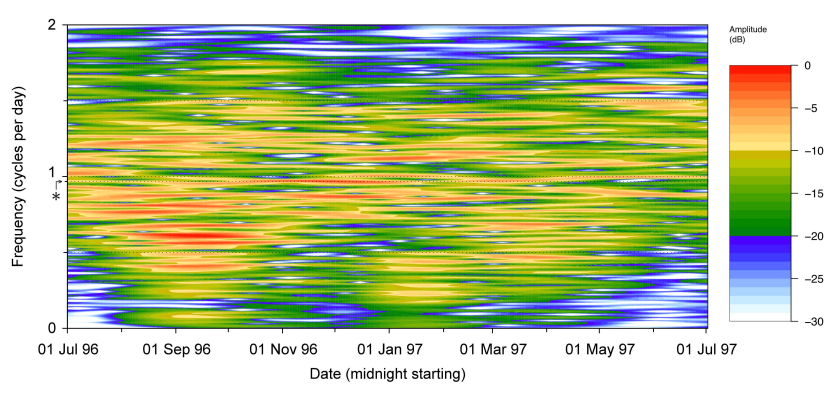

Fig. 4. EMD, Sur-Frêtes, IMF 4; frequencies grouped around 1 cycle per day (the asterisk indicates the M1 tidal harmonic frequency).

For the Syabru-Bensi time-series, SSA revealed six significant components, of which the first two comprise major (nonstationary) features of the data and the third to sixth contain frequency spectra centred at 1 and 2 cycles per day.

Figure 6 shows the spectrogram for Sur-Frêtes RCs 3,4 combined. Figure 7 shows the spectrogram for Syabru-Bensi

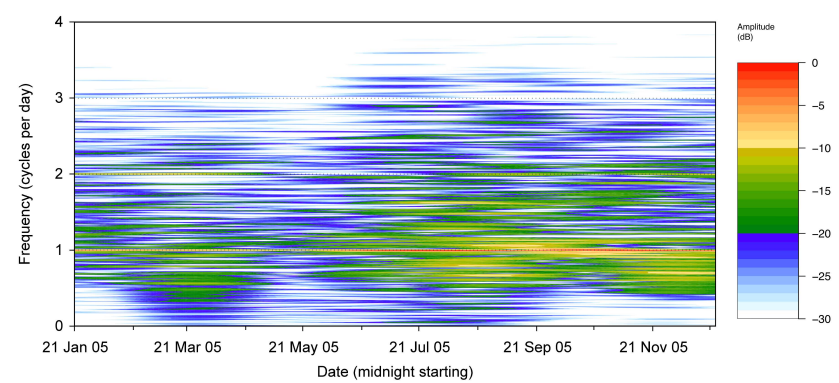

Fig. 5. EMD, Syabru-Bensi, IMFs 3,4 combined; frequencies grouped around 1 and 2 cycles per day.

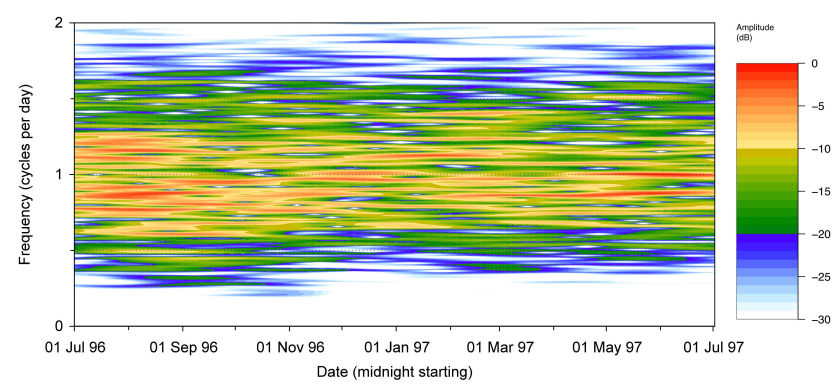

Fig. 6. SSA, Sur-Frêtes, RCs 3,4; frequencies grouped around 1 cycle per day.

RCs 3-6 combined. The results are also summarised in Table 1.

Reference to the figures shows that the frequency components are more tightly centred about 1 and 2 cycles per day for the Syabru-Bensi data than for the Sur-Frêtes data. In both time-series, EMD and SSA yield comparable results, although the comparison is more complete for the Syabru- 


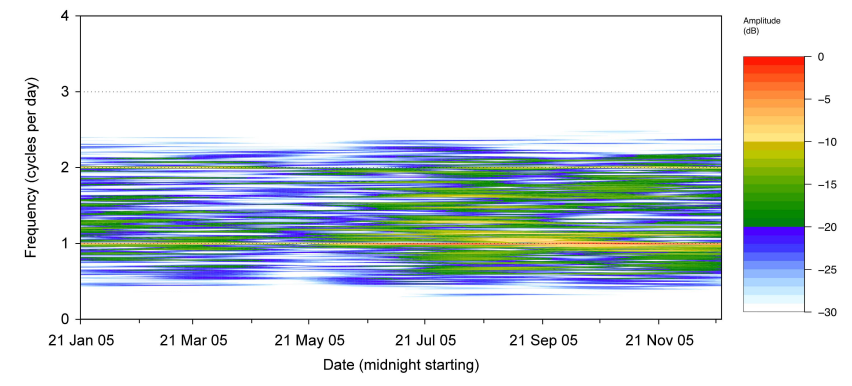

Fig. 7. SSA, Syabru-Bensi, RCs 3-6 combined; frequencies grouped around 1 and 2 cycles per day.

Bensi data. For the Sur-Frêtes data, EMD reveals both 24-h, S1, and 12-h, S2, tidal components whereas SSA reveals only a 24-h, S1, component. However, for the Syabru-Bensi data, both EMD and SSA reveal 24-h, S1, components and weaker 12-h, S2, components. These components are attributed to barometric tides (Haurwitz and Cowley, 1973; Richon et al., 2009).

In addition, EMD reveals frequency components consistent with the presence of luni-solar tidal cycles in the SurFrêtes data. Inspection of Fig. 3 reveals an approximately two-month period centred on 1 December where there is a clear 24.8-h, M1, tidal component. This is accompanied by weaker evidence consistent with a 12.4-h, M2, component in Fig. 2. Despite the presence of other spectral components in the "noise" of the spectrograms, there is no evidence consistent with other diurnal or semi-diurnal luni-solar tidal components in either time-series.

\section{Discussion and conclusions}

EMD has revealed "noisy" sets of diurnal and semi-diurnal frequency components in both time-series. Both time-series contain S1 (24-h) and S2 (12-h) solar tidal harmonics but these are much more clearly defined in the Syabru-Bensi data than in the Sur-Frêtes data. In addition, the Sur-Frêtes data contain intermittent but clear spectral components consistent with the presence of the M1 (24.83-h) and weaker evidence consistent with the presence of the M2 (12.42-h) luni-solar tidal harmonics, evidence not present in the Syabru-Bensi data. SSA clearly confirms the presence of both the S1 and S2 harmonics identified by EMD in the Syabru-Bensi data. SSA of the Sur-Frêtes data clearly confirms the presence of the S1 harmonic identified by EMD, but not of the S2 harmonic. In both time-series but particularly Sur-Frêtes data, the interpretation of the singular spectrum and hence the frequency spectra of the reconstructed components is complicated by the large number of closely-spaced frequencies as revealed by EMD.
Neither time-series contains 7-day components: thus, whilst anthropogenic influences cannot be discounted, these are less likely than if 7-day components were present. Consequently, the presence of the S1 and S2 cycles is attributed to the barometric diurnal and semi-diurnal tides, there being no known anthropogenic influences at either location. The presence of the intermittent M1 and M2 cycles in the SurFrêtes data is primarily attributed to luni-solar diurnal and semi-diurnal earth tides, any effects of ocean tidal-loading being weak at this location, $600-700 \mathrm{~km}$ from France's Atlantic coast and the Mediterranean having small tides. The intermittency of all the cyclic features is attributed to nonstationary variations in soil properties.

This investigation has revealed hitherto unobserved periodic variations in radon time-series and demonstrates the potential for the observation and analysis of radon time-series to reveal information regarding crustal and surficial structures and processes. This complements the previous observation of lunar-monthly and bi-weekly tidal cycles in radon data (Crockett et al., 2006a).

Such information is necessary to characterise the physical processes controlling the radon time series, affected by numerous and intermingled effects. While S1 and S2 components suggest temperature and atmospheric pressure effects, M1 and M2 tidal components suggest earth-tide effects and, thus, some sensitivity of radon concentration to large scale crustal deformation. Such sensitivity needs further confirmation, in Sur-Frêtes and in other sites, but might help in understanding possible radon earthquake precursors (Crockett et al., 2006b; Crockett and Gillmore, 2010).

Acknowledgements. The authors thank their colleagues in both research groups for their continuing support assistance throughout this research. This is IPGP contribution 2622.

Edited by: G. Gillmore

Reviewed by: S. M. Barbosa and another anonymous referee

\section{References}

Crockett, R. G. M., Gillmore, G. K., Phillips, P. S., Denman, A. R., and Groves-Kirkby, C. J.: Tidal Synchronicity of BuiltEnvironment Radon Levels in the UK, Geophys. Res. Lett., 33, L05308, doi:10.1029/2005GL024950, 2006a.

Crockett, R. G. M., Gillmore, G. K., Phillips, P. S., Denman, A. R., and Groves-Kirkby, C. J.: Radon Anomalies Preceding Earthquakes Which Occurred in the UK, in Summer and Autumn 2002, Sci. Total Environ., 364, 138-148, 2006 b.

Crockett, R. G. M. and Gillmore, G. K.: Spectral-Decomposition Techniques for the Identification of Radon Anomalies Temporally Associated with Earthquakes Occurring in the UK in 2002 and 2008, Nat. Hazards Earth Syst. Sci., in preparation, 2010.

Ghil, M., Allen, M. R., Dettinger, M. D., Ide, K., Kondrashov, D., Mann, M. E., Robertson, A. W., Saunders, A., Tian, V., Varadi, F., and Yiou, P.: Advanced Spectral Methods For Climatic Time Series, Rev. Geophys., 40, 3.1-3.41. doi:10.1029/2001RG000092, 2002. 
Haurwitz, B. and Cowley, A. D.: The Diurnal and Semidiurnal Barometric Oscillations, Global Distribution and Annual Variation, Pure Appl. Geophys., 102, 192-222, 1973.

Huang, N. E., Shen, Z., Long, S. R., Wu, M. L., Shih, H. H., Zheng, Q., Yen, N. C., Tung, C. C., and Liu, H. H.: The Empirical Mode Decomposition and Hilbert Spectrum for Nonlinear and Nonstationary Time Series Analysis, Proc. Roy. Soc. A, 454, 903-995, 1998.

Miles, J. C. H.: Temporal variation of radon levels in houses and implications for radon measurement strategies, Radiat. Prot. Dosim., 93, 369-375, 2001.

Neves, L. J. P. F., Barbosa, S. M., and Pereira, A. J. S. C.: Indoor Radon Periodicities and their Physical Constraints: a study in the Coimbra region (Central Portugal), J. Environ. Radioactivi., 100, 896-904, doi:10.1016/j.jenvrad.2009.06.017, 2009.

Perrier, F., Richon, P., and Sabroux, J.-C.: Temporal Variations Of Radon Concentration in The Saturated Soil of Alpine Grassland: the role of groundwater flow, Sci. Total Environ., 407, 23612371, 2009a.
Perrier, F., Richon, P., Byrdina, S., France-Lanord, C., Rajaure, S., Koirala, B. P., Shrestha, P. L., Gautam, U. P., Tiwari, D. R., Revil, A., Bollinger, L., Contraires, S., Bureau, S., and Sapkota, S. N.: A direct evidence for high carbon dioxide and radon-222 discharge in Central Nepal, Earth Planet. Sci. Lett., 278, 198-207, 2009b.

Richon, P., Perrier, F., Pili, E., and Sabroux, J.-C.: Detectability and Significance of $12 \mathrm{Hr}$ Barometric Tide in Radon-222 Signal, Dripwater Flow Rate, Air Temperature and Carbon Dioxide Concentration in an Underground Tunnel, Geophys. J. Int., 176, 683-694 doi:10.1111/j.1365-246X.2008.04000, 2009.

Vautard, R. and Ghil, M.: Singular Spectrum Analysis In Nonlinear Dynamics, With Applications To Paleoclimatic Time Series, Physica D, 35, 395-424, 1999. 